\title{
Changes in cortical bone channels network and osteocyte organization after the use of zoledronic acid
}

Gustavo Davi Rabelo 1,2, Bruno Augusto Nassif Travençolo, Marcio Augusto Oliveira', Marcelo Emílio Beletti', Marina Gallottini', Fernando Ricardo Xavier da Silveira'

\begin{abstract}
Objective: The aim of this study was to evaluate the effects of zoledronic acid (ZA) on the cortical bone channels network (CBCN) and osteocyte organization in relation to the bone channels. Materials and methods: Eighteen male Wistar rats were divided into control (CG) and test groups (TG). Twelve animals from TG received 3 ZA doses $(7.5 \mu \mathrm{g} / \mathrm{kg})$, and 6 animals from CG did not receive any medication. TG animals were euthanized at $14(n=6)$ and $75(n=6)$ days after drug injection. CBCN was analyzed in mandibles and tibias using computational routines. The osteocyte organization was qualitatively evaluated in tibias using a three-dimensional reconstruction of images from serial histological sections. Results: Significant differences in CBCN of tibia were found between the treated and untreated rats, with a wider range of sizes and shapes of the channels after the use of ZA (channels area $p=0.0063$, channels area $S D p=0.0276$ ) and less bone matrix (bone volume $p=0.0388$ ). The alterations in the channels' morphology were more evident at 75 days after the drug injection (channels perimeter $p=0.0286$ ). No differences were found in mandibles CBCN. The osteocyte distribution revealed more variable patterns of cell distribution in ZA groups, with non-homogeneous distribution of cells in relation to the bone channels. Conclusion: Zoledronic acid induces structural changes in $\mathrm{CBCN}$ and modifies the osteocyte arrangement in cortical bone in the tibia; also, the variability in the morphology of bone channels became more evident after a certain time of the use of the drug. Arch Endocrinol Metab. 2015;59(6):507-14
\end{abstract}

Keywords

Bone; bone channels; osteocyte; zoledronic acid; cortical bone
1 Departamento de Estomatologia, Faculdade de Odontologia, Universidade de São Paulo (USP), São Paulo, SP, Brazil ${ }^{2}$ A.C. Camargo Cancer Center, São Paulo, SP, Brazil

${ }^{3}$ Faculdade de Computação, Universidade Federal de Uberlândia (UFU), Uberlândia, MG, Brazil ${ }^{4}$ Instituto de Ciências Biomédicas, UFU, Uberlândia, MG, Brazil

Correspondence to: Gustavo Davi Rabelo

Universidade de São Paulo Faculdade de Odontologia, Departamento de Estomatologia Av. Prof. Lineu Prestes, 2227 05508-000 - São Paulo, SP, Brazil drgustavorabelo@yahoo.com.br

Received on June/19/2015 Accepted on July/6/2015

DOI: 10.1590/2359-3997000000097

\section{INTRODUCTION}

B isphosphonates (BP) have become the standard class of drugs for treating patients with osteoporosis and in preventing and treating skeletal complications in patients with cancer ( 1 ). Intravenous (IV) zoledronic acid (ZA), a commonly used BP, has been classified as a heterocyclic nitrogen-containing bisphosphonate that potently inhibits osteoclastic bone resorption in various short-term in vitro and in vivo pharmacological screening models (2). Once internalized by osteoclasts, ZA inhibits the mevalonate pathway, which stops the cell function and slows resorption, interfering in bone metabolism. In addition, other mechanisms were already related with the action of $\mathrm{ZA}$ - for example, antiangiogenic and some immunomodulatory effects (3).

Several studies have assessed the effects of ZA on bone properties, including biochemical characteristics, remodeling rates, structural changes, and biomechanical features. Studies evaluating cortical bone character- istics revealed dose- and timing-dependent effects on turnover, microstructure, and mechanical properties (4-7). Concerning microarchitecture, it is known that bone multicellular units are changed, and together with an anti-angiogenic effect, the zoledronic acid could cause a decrease (or complete loss of) of intraosseous vascularity (1).

The question of whether the characteristics and location of cells and channels have an influence on bone response to changes remains unresolved. Cortical porosity has been demonstrated to be a major determinant of strength, stiffness, and fracture toughness; also, pore structure and distribution produce heterogeneous local strain and stress fields within the cortical bone (8). Thus, the organization of bone channels is continuously modified in order to enhance the mechanical strength of the bone structure in front of the imposed solicitations (9).

The regulation of bone formation by osteocytes, as inhibiting or activating the osteoblasts, is coordinated 
by a complex interaction between bone cells, and the function of the osteocytes will depend on their spatial position (10). It is well known that there is a relationship between bone properties and the distribution of its components, principally concerning microarchitecture (11). Regarding osteocytes distribution in the matrix and their relation with the vascular channels, there is a communication system between cells-cells and cellschannels characterized as a network grid between osteocytes and their process, radiated through canaliculi to other surrounding osteocytes and channels. It is a system in which the load-induced fluid flow occurs through the canaliculi, and allows the exchange of this fluid and molecules between canaliculi and osteocytes lacunae, as well as with the channels $(12,13)$. With some changes in microarchitecture, and with consequent spatial changes in the channels and osteocytes location, this communication system could be compromised (14).

Possible variation in bone tissue metabolism, for example, after the use of the ZA, could be identified in microarchitecture changes never analyzed before. The spatial distribution of bone elements, i.e. cells and channels, should be an important tool for revealing an altered or a more organized and developed microenvironment. In this way, the aim of this study was to evaluate the cortical bone channels network and the osteocyte distribution in mandible and tibia, after the use of zoledronic acid.

\section{MATERIALS AND METHODS}

\section{Animals and drug administration}

Eighteen male Wistar rats (Rattus norvegicus) (average weight of $424 \pm 103 \mathrm{~g}$, average age of $6.5 \pm 2.7$ months) were divided randomly into 2 groups: control $(\mathrm{CG}, \mathrm{n}=6)$ and test (TG, $\mathrm{n}=12$ ) groups. The TG received (subcutaneously) a $7.5 \mu \mathrm{g} / \mathrm{kg}$ dose of zoledronic acid $\left(\right.$ Novartis ${ }^{\circledR}$ ) once a week, during three weeks, as in the study of Sonis and cols. (15). The animals in TG were euthanized at 14 days $(\mathrm{ZAl} 4 \mathrm{D}, \mathrm{n}=6)$ and 75 days $(\mathrm{ZA75D}, \mathrm{n}=6)$, after the last drug injection. The control group did not receive any medication and were euthanized at the same time as the TG animals (three as in ZAl4D and three as in ZA75D).

All animals were maintained with food and water ad libitum, and the Institutional Animal Care and Use Committee of the Institute of Biomedical Sciences at the University of São Paulo approved all procedures (4lp100b2). The experimental procedures and care of the animals were made in accordance with the European Convention for the Protection of Vertebrate Animals used for Experimental and Other Scientific Purposes.

All animals had their mandible and tibia dissected, and segments were sectioned and placed in $10 \%$ formaldehyde buffer for 72 hours. The mandible and tibia were decalcified in 4\% EDTA for 4 months and embedded in paraffin. The tibias were cut transversally, and the central part of the diaphysis was used in the study. For the mandibles, a coronal plane was chosen for sectioning, and the inclusion plane was defined in a position where it is possible to identify the root of the molar tooth.

\section{Bone microarchitecture assessment - Analysis of cortical bone channels network}

For each sample, three nonserial thin sections with 3 $\mu \mathrm{m}$ thickness were obtained and stained with hematoxilin and eosin (HE) (Figure 1A). All of the histological sections were analyzed through digitized images obtained with a microscope coupled to a camera connected to a computer (AxioVision, Zeiss). The images were captured with the objective lens of $40 \mathrm{X}$ as follows: for the mandible, one image was captured in the superior cortical (surrounding the root of the first molar), and the other image was captured in the inferior cortical part (vicinity of the incisor teeth posterior area within the mandible); in the tibia, one image was captured in a random cortical area. This procedure resulted in a total of 108 histological images of the mandible and 54 images of the tibia.

The images were segmented and analyzed using a previous described methodology by Rabelo and cols. (16) and proposed by Oliveira and cols. (17), which consists of an interactive method of drawing and selecting all channels (vascular areas as Havers and Volkmann channels) in the image (Figure 1B). Afterward, an analysis was performed by algorithms developed in the SCILAB mathematical environment, considering several morphological features of cortical bone channels network $(\mathrm{CBCN})$ and using image texture characterization. Each image was analyzed separately; after selecting the bone channels, they were called objects of interest $(\mathrm{OI})$, and new images were created containing only the OI (Figure 1B). The bone volume and OI features were analyzed according to the following variables: $\mathrm{BV} / \mathrm{TV}$, total bone volume per tissue volume (percentage of bone 
matrix - amount of black pixels in the image); mean and standard deviation of the OI areas (the number of pixels inside the OI); mean and standard deviation of the OI perimeters (the sum of the distance between pixels along each OI border); and lacunarity (18).

\section{Three dimensional (3D) osteocyte organization}

Osteocyte organization was evaluated in 3D considering only 2 samples in the control group and 4 in the test group (ZAl4D and ZA75D, $\mathrm{n}=2$ each). In order to obtain the 3D data, histological sequential images derived from 20 serial sections with $3 \mu \mathrm{m}$ thickness each were aligned and grouped, forming a volume (Figure 1A). All samples were processed according to the technique proposed by Travençolo and cols. (19). The main idea of the methodology is to determine the distribution of the distances between objects and a reference structure. In particular, we were interested in studying the spatial distribution of the osteocytes lacunae (the objects) in relation to de blood vessels (the reference structure).

In the first step of the methodology, the images were manually segmented in order to identify the channels containing the blood vessels (Figure $1 \mathrm{~B}$ ) and osteocyte lacunae (Figure $1 \mathrm{C}$ ). Then, the $3 \mathrm{D}$ reconstruction of the sample and the computation of the spatial coordinates of the osteocytes and channels were performed (Figure 1D). The distance transform (18) relative to the channels was determined - i.e., a map of distances in which is possible to know the distance of any point in the $3 \mathrm{D}$ space to the closest channel. With this map we can determine the distance between an osteocyte and its closest channel. This computation method was performed for each osteocyte in the image. As a result, each cell was characterized with a number value that indicates its distance to the closest channel. From these values of distances, it was possible to create regions surrounding each channel, automatically delimited in the $3 \mathrm{D}$ image by the program, considering the spatial localization of the osteocytes surrounding the channels. These regions can be seen as gray light surfaces around the channels (Figure 1D) and were called isodistances. In this way, the nearest isodistance to the channel was called isodistance 1 and so on successively, to the longest isodistance, called isodistance 6 .
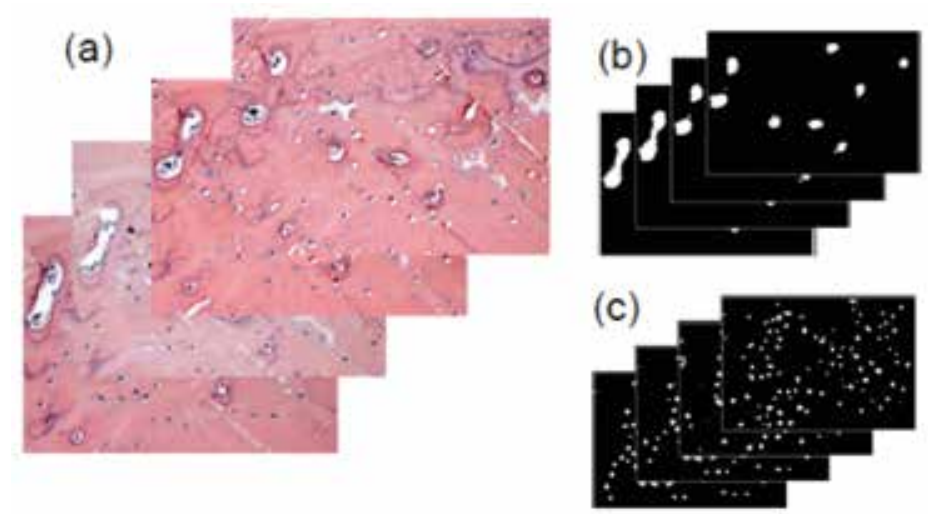

(d)

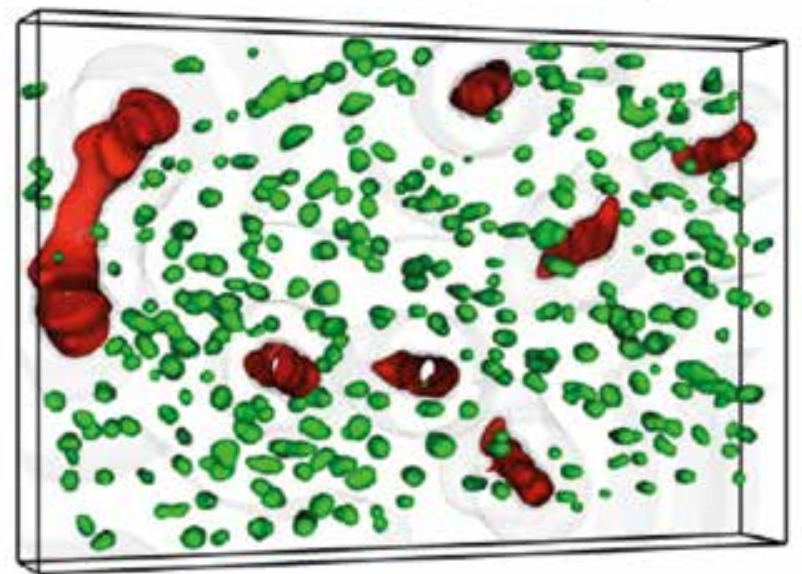

Figure 1. Three-dimensional reconstruction (3D) of tibia cortical bone. (A) Illustration of the original serial sections aligned in order to build a 3D volume. (B) Result of the manual segmentation of the images to identify the channels. (C) Segmentation of the osteocytes. (D) 3D reconstruction of the channels, evidenced in red, and the osteocytes, shown in green. The light gray surfaces surrounding the channels represent the isodistances surfaces. 
After the establishment of the isodistances, the distribution of the osteocytes was evaluated according to the isodistance in which it is located - i.e., for each isodistance the number of osteocytes located inside the region represented by the isodistance is computed. A plot was created to show how the osteocytes were organized in relation to each channel in the image (Figure 2), considering the isodistances 1 to 6 . If a curve subtly changed, for example with a big decline, it was assumed that the osteocytes organization surrounding the channels in that image was more unorganized. On the other hand, if the curve presented low variability, represented by a plot as straight as possible, it was assumed that the osteocyte organization was more homogeneous and organized, as there was no higher variation in the number of osteocytes in relation to each channel, as with the control samples in figure 2 .

\section{Statistical analysis}

Data were initially evaluated using the Kolmogorov -Smirnov test, which was used to assess the normality of the data distribution, and then analyzed using Kruskal-Wallis test (not assuming Gaussian distribution). In order to identify the difference between groups when there was a significant statistical diffe- rence, the Dunn's post-test was performed (error probability $5 \%$ ).

\section{RESULTS}

The histological analysis in all animals revealed the presence of cortical bone with Haversian channels and osteocytes, with the bone channels network revealing specific characteristics in the different types of bone. Comparing the bone channels network characteristics of the mandible in the control and test groups, no statistically significant difference was found in any of the features evaluated (Table 1).

However, evaluation of the bone channels network in the tibias revealed significant differences in some parameters analyzed. After administration of ZA, the bone channels structure changed the topography of the network, revealing channels with higher values of OI area and OI area standard deviation in both TG subgroups (ZAl4D and ZA75D) (Table 1). Different shapes of the channels were identified as well, with higher values of OI perimeter. Interestingly, the OI perimeter was different only between the control group and the ZA75D. A lower BV/TV was also found when comparing the control group with ZA75D (Table 1).

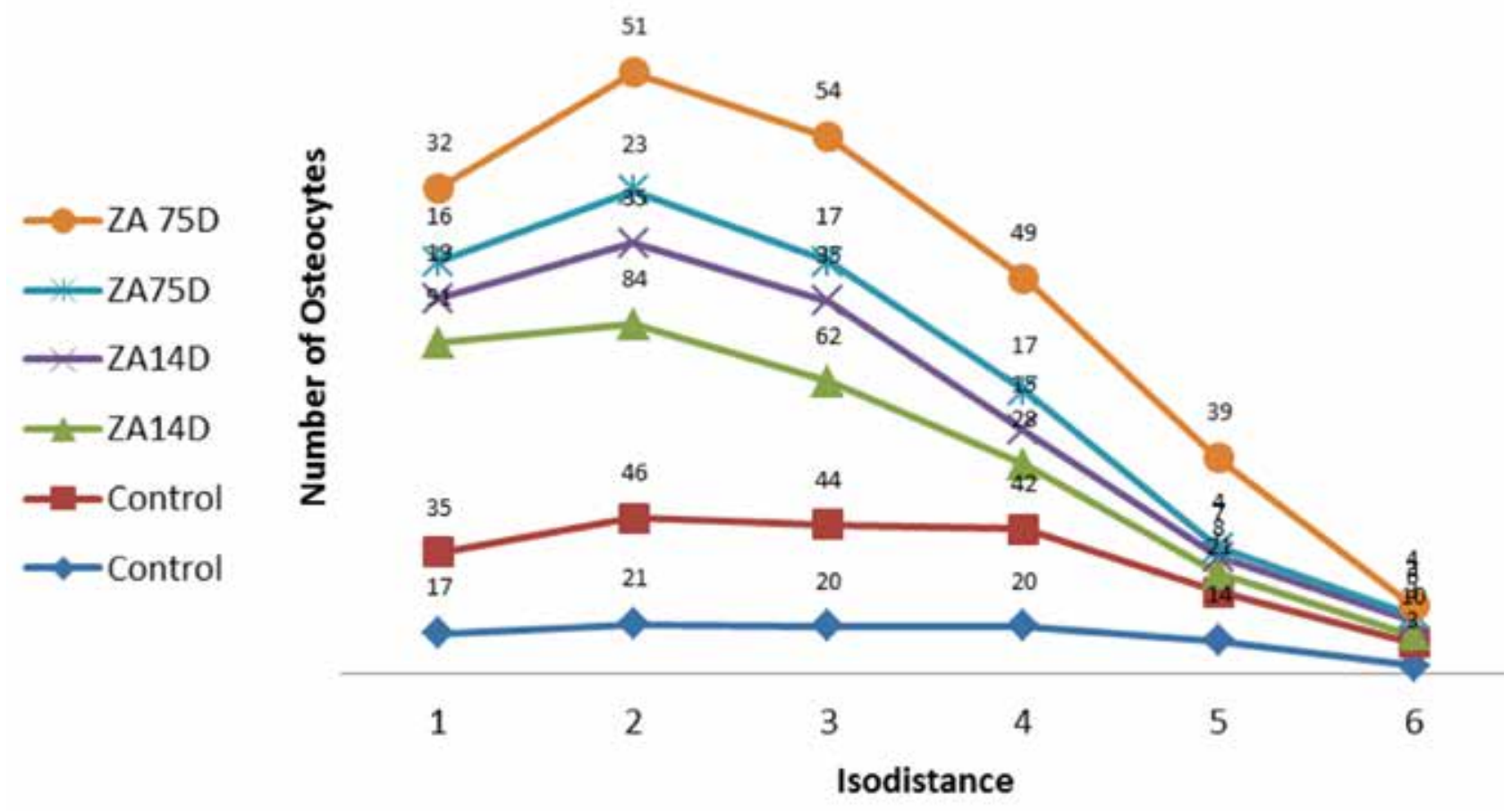

Figure 2. The plot represents the number of osteocytes in relation to the increasing in distance (isodistances) from the bone channels. Control - control group; ZA - zoledronic acid group; 14D - sacrificed fourteen days after drug injection; 75D - sacrificed seventy five days after drug injection. All analyses were performed on the tibia. Each line represents a rat of the indicated treatment/control group. 
Concerning lacunarity, there were no significant differences between the groups (Table 1).

The $3 \mathrm{D}$ technique revealed an interesting view of the microstructure of the bone tissue, with osteocyte and channels distributed spatially in the matrix. In a qualitative analysis, it was possible to identify the spatial position of bone components and an existing relationship of the cells in relation to the bone channels
(Figure 1D). The distribution of the osteocytes along the periphery of the Haversian channels allowed for an assumption that there is a more homogeneous/heterogeneous connectivity between lacunae and the bone channels with/without the use of zoledronic acid, respectively. The difference in the osteocyte distribution along the different isodistances between control and test group is exemplified in figure 3 .

Table 1. Bone volume, object of interest (OI) area and perimeter, standard deviation (SD) and lacunarity

\begin{tabular}{|c|c|c|c|c|c|c|}
\hline & \multicolumn{3}{|c|}{ Mandible } & \multicolumn{3}{|c|}{ Tibia } \\
\hline & Control & ZA14D & ZA75D & Control & ZA14D & ZA75D \\
\hline BV/TV (\%) & $95.20(3.545)$ & 95.53 (3.216) & 95.66 (2.715) & $96.81(1.674)^{\mathrm{a}}$ & $95.27(3.349)^{a, b}$ & $94.52(2.968)^{b}$ \\
\hline$p$ & \multicolumn{3}{|c|}{0.9548} & \multicolumn{3}{|c|}{$0.0388^{*}$} \\
\hline Ol area & 3027 (2699) & 2688 (1816) & 2862 (1500) & $1107(365.5)^{b}$ & $1787(840.3)^{\mathrm{a}}$ & $1853(962.7)^{\mathrm{a}}$ \\
\hline$p$ & \multicolumn{3}{|c|}{0.5452} & \multicolumn{3}{|c|}{$0.0063^{*}$} \\
\hline Ol area SD & 3175 (3544) & 2821 (2676) & 2494 (1980) & 1010 (527.4) & $1813(1276)$ & 2236 (2116) \\
\hline$p$ & \multicolumn{3}{|c|}{0.9244} & \multicolumn{3}{|c|}{$0.0276^{*}$} \\
\hline Ol perimeter & $272.1(114.4)$ & 248.4 (88.38) & 274.2 (71.32) & $193.5(48.19)^{b}$ & $236.6(69.71)^{a, b}$ & $264.3(99.28)^{\mathrm{a}}$ \\
\hline$p$ & \multicolumn{3}{|c|}{0.4547} & \multicolumn{3}{|c|}{$0.0286^{\star}$} \\
\hline Ol perimeter SD & $166.4(121.7)$ & 131.7 (89.15) & 126.5 (79.61) & $112.4(48.51)$ & 162.1 (78.12) & $236.1(235.0)$ \\
\hline$p$ & \multicolumn{3}{|c|}{0.3729} & \multicolumn{3}{|c|}{0.1002} \\
\hline Lacunarity & $10.00(5.523)$ & 9.581 (5.239) & $11.45(4.303)$ & 13.11 (3.924) & $11.08(4.166)$ & 10.06 (3.969) \\
\hline$p$ & \multicolumn{3}{|c|}{0.4331} & \multicolumn{3}{|c|}{0.0501} \\
\hline
\end{tabular}

* Kruskal-Wallis test: significant differences $(\mathrm{p}<0.05)$. BV/TV bone volume. ZA Zoledronic Acid. 14D animals sacrificed 14 days after drug injection. 75D animals sacrificed 75 days after drug injection.

a,b Different superscript letters indicate significant differences by Dunns post-test $(\mathrm{p}<0.05)$. Control group: three animals were sacrificed at same time as ZA14D group and three sacrificed at same time as ZA75D group.

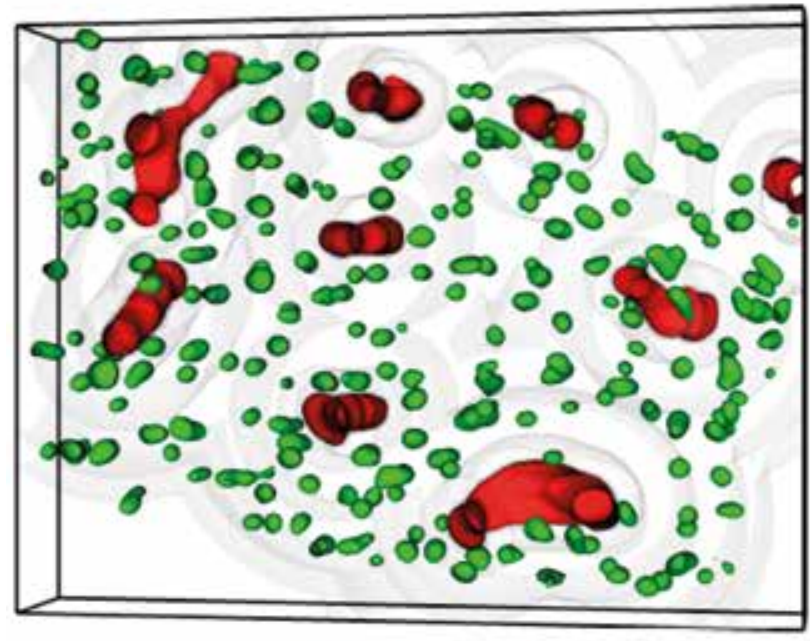

A) Control

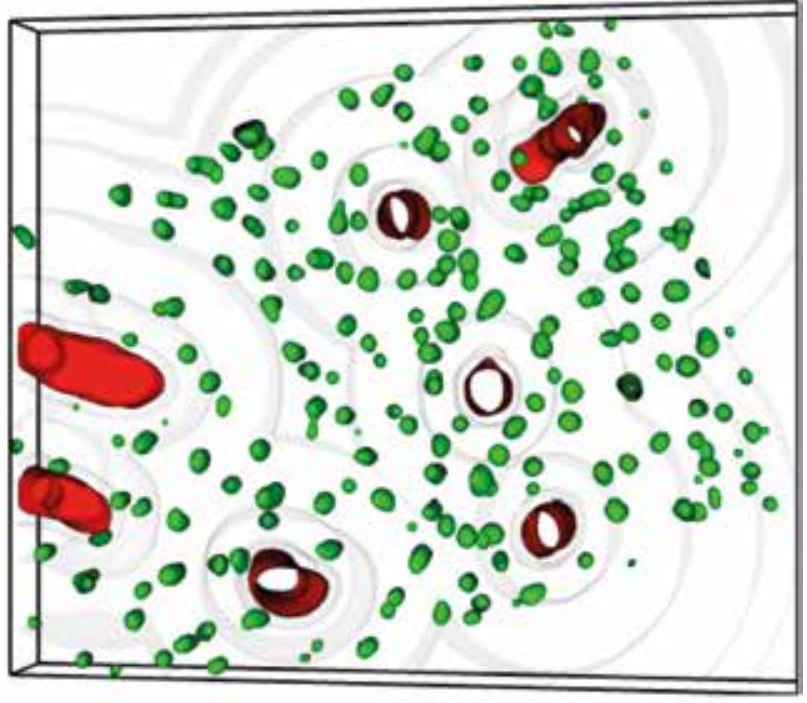

B) Test

Figure 3. 3D Evaluation - Bone channels in red, osteocytes in green, isodistances in gray. (A) Control Group; (B) Test group. 
The differences in the organization of the osteocytes between control and ZA groups were observed in 3D. The osteocyte lacunae distribution in the control group presented more homogeneity in its distribution in relation to channels, i.e., more homogeneous osteocyte organization. It was possible to ascertain a decrease in the number of cells noted at longer distances from the channels in a gradually decreasing manner (Figure 2). In order to facilitate the visualization of osteocytes distribution in relation to the channels and their decrease in relation to the longest isodistances, this semi-quantitative analysis was drawn in the plot presented in figure 2 , making it possible to measure the variation in the number of cells around the channels for each isodistance in each sample. In the ZA groups, the osteocyte distribution showed the highest number of osteocytes in the first isodistances (Isodistances 1 and 2; Figure 2), followed by an increasing decline (Isodistances 3, 4, 5 , and 6; Figure 2); on the other hand, in the control group, this high variability was not clearly evident, and the lines formed in these samples were straighter than those of the TG.

\section{DISCUSSION}

Microarchitectural changes in bone after treatment with bisphosphonates, especially zoledronic acid, have been studied in different ways, but particularly in the field of osteocyte distribution and their relationship with the bone channels, there are little data in the literature. One of the main findings in this study was that the difference between the control and ZA groups in relation to cortical bone channels topography was significant only in the tibia, and not in the mandible. Indeed, the most important result was that the $\mathrm{ZA}$ is able to change the channels topography in the tibial cortical bone. Also, some of the differences in the parameters analyzed were evident only after a longer period of time after the use of the drug, in the case of the ZA75D group. All these findings provides information about the changes in bone channels morphology, with reference to the variability in their sizes and shapes, suggesting wider and deformed channels composing the bone channels network in a long bone after the use of zoledronate. Bernhard and cols. (10) found similar results with higher values of Haversian canal diameter and Haversian canal area in the mid-cortex between aged and young women, and those with osteoporosis and those treated with bisphosphonates. However, the- se two parameters did not show a significant intergroup difference after statistical correction.

Although ZA was previously related to changes in trabecular microarchitecture, our results also revealed evidence of changes in the cortical part. With bigger channels and less matrix, it was assumed that the cortical bone presented with lower bone volume and the presence of wider channels, suggesting an increase in cortical porosity. Zebaze and cols. (20), evaluating aging effects on bone in the radius and femurs of women, concluded that most bone loss is cortical, not trabecular, and occurs by intracortical rather than endocortical or trabecular remodeling. Further, the identification of the mechanisms causing intracortical remodeling is likely to improve the understanding of the pathogenesis of bone fragility. In this way, alterations on cortices after the use of ZA might compromise bone properties such as stiffness and flexibility. In addition, it is wellestablished that ZA suppresses bone formation and decrease bone turnover $(21,22)$, facts that could also alter the porosity. Therefore, it has to be mentioned that the responses to ZA present different magnitudes across different bone sites (23), and different studies in different conditions should reveal different results related to bone channels and cortical porosity.

There was an agreement with the results of the $\mathrm{CBCN}$ evaluation and the results of $3 \mathrm{D}$ analysis. With the osteocyte organization being more unorganized, and the channels altered in their morphology, it was assumed that in the situations with high variability of cell distribution in relation to the channels in osteons, the communication between channels-lacunae and lacunae-lacunae could be compromised in some way, a fact that could influence how fluid flows through these communication system and compromise the good bone function. In some way, a compromised bone function and the increase in porosity could be related.

After the use of $\mathrm{ZA}$, the $3 \mathrm{D}$ analysis revealed that the number of the osteocytes decreases in relation to the increase in distance from channels. Indeed, this decrease turns out to be different between CG and TG, with higher variation in number of cells per isodistance in the ZA subgroups (Figure 2). These results points to an inconstancy in cell distribution around the channels, possibly reflecting some changes that occurred in the $\mathrm{CBCN}$ and consequently in the cell array. As reported by Bernhard and cols. (10), rudimentary osteocytic networks may lead to a delayed and/or hampered repair mechanisms and subsequent bone fragility. How- 
ever, it must be considered that in our study, the osteocyte lacunar density was not evaluated. In addition, one major point is the $3 \mathrm{D}$ analysis provided topological information about the osteons that allowed to identify specific changes in each sample analyzed, characterizing an individual analysis.

The disruption of the homogeneity in osteocytes distribution in the matrix, with longer distances from cell to cell, can affect lacunocanalicular system-dependent events, as for example the mechanotransduction and fluid flow. Previous studies reported that this system mechanism can dictate the mechanotransduction and functional adaptation of bone (24). Additionally, the fluid pressures in this system are associated with deformation-induced pressures in the Haversian system (25).

It is important to emphasize that the $3 \mathrm{D}$ analysis was done qualitatively and semi-quantitatively through the distribution of osteocytes, and a reduction in their number relative to the increase in the distance from channels was evident. Previous studies reported that some anatomical changes and different skeletal sites present distinct compositions concerning the structural and cellularity fields. Viana and cols. (9) reported that the bone network is partitioned into communities or modules and that each community exhibited distinct topological properties that are possibly linked with their specific functions in their different sites. HimenoAndo and cols. (26) showed that the number of cellular processes and their bifurcation points per osteocyte in the tibias were significantly higher than those in the parietal bone, with morphometric data of osteocyte network revealing more endpoints, branching points, and segments in the tibia. Combined with our results, it was suggested that the cellularity and the $\mathrm{CBCN}$ is different when comparing different sites as mandible and tibia. In this way, each site should be evaluated considering its specific characteristics.

In relation to the mandible evaluation, the changes in the topography and microstructure were inconspicuous, suggesting that the drug does not necessarily interfere in the bone channels' characteristics at this bone site. Clearly, the results of the mandible in both areas did not show significant differences between control and test groups. One situation that could explain these findings is that the mandible could be more resilient to changes. If $\mathrm{ZA}$, that clearly affect bone turnover, do not change the channels in the mandible, it was assumed that the drug may have a different effect in this bone site.
Studies developed to identify changes in the jaws after the use of ZA revealed several alterations in bone architecture and turnover. Nevertheless, those changes were related with higher doses, longer periods of drug administration, and concomitance with other procedures. Senel and cols. (27) found some differences in the mandible after ZA treatment, with inflammatory cell infiltration along with polymorphonuclear leukocytes leading to an abscess appearing in the gingival epithelium and extending into the posterior mandibular alveolar bone. They also found empty osteocyte lacunae in some cases. However, the authors administered ZA for periods of 6 and 8 weeks, 3 times a week at a proportion of $0.1 \mathrm{mg} / \mathrm{kg}$. Sonis and cols. (15) described bony changes in the jaws similar to osteonecrosis after the use of ZA at a similar dose of $7.5 \mu \mathrm{g} / \mathrm{kg}$; however, these changes were found in animals that received ZA plus dexamethasone and were then submitted to dental extractions. In this way, the bisphosphonate-related osteonecrosis of the jaws, considered a multifactorial side effect, may have one of the etiology factors as the relation between the concomitant use of ZA and corticoids, and also with dental manipulation, acting as a triggering factor, pointing to the multifactorial etiology of this side effect. At this point, the lack of alterations in the mandible revealed in our study reflect that the use of ZA without others predisposing factors was not sufficient to explain the etiology of the osteonecrosis.

Considering all the data found in the histological analysis, together with the 3D evaluation of osteons, it became clear that in the tibias of CG the channels revealed some similarity in their morphology and the osteocyte distribution was more homogeneous along the isodistances. On the other hand, in ZA groups, the channels became modified after some period of the use of the drug and the CBCN became altered, also, with a more unorganized osteocyte distribution in the long bone. In conclusion, zoledronic acid induces structural changes in the cortical bone channels network in the tibias, but does not modify the morphology of channels in mandibles. In long bones, the cortical channels became wider and present some variations in their shapes, with a decrease in bone volume. The osteocyte organization in relation to the channels was more variable and less organized after the use of drug.

Disclosure: no potential conflict of interest relevant to this article was reported. 


\section{REFERENCES}

1. Migliorati CA, Epstein JB, Abt E, Berenson JR. Osteonecrosis of the jaw and bisphosphonates in cancer: a narrative review. Nat Rev Endocrinol. 2011;7(1):34-42.

2. Hornby SB, Evans GP, Hornby SL, Pataki A, Glatt M, Green JR. Long-term zoledronic acid treatment increases bone structure and mechanical strength of long bones of ovariectomized adult rats. CalcifTissue Int. 2003;72(4):519-27.

3. Young RJ, Coleman RE: Zoledronic acid to prevent and treat cancer metastasis: new prospects for an old drug. Future Oncol. 2013;9(5):633-43.

4. Allen MR. The effects of bisphosphonates on jaw bone remodeling, tissue properties, and extraction healing. Odontology. 2011;99(1):8-17.

5. Bilston LE, Little DG, Smith NC, Williams P, Briody J. Zoledronic acid improves the mechanical properties of normal and healing bone. Clin Biomech (Bristol, Avon). 2002;17(9-10):716-8.

6. Day JS, Ding M, Bednarz P, van der Linden JC, Mashiba T, Hirano $\mathrm{T}$, et al. Bisphosphonate treatment affects trabecular bone apparent modulus through micro-architecture rather than matrix properties. J Orthop Res. 2004 May;22(3):465-71.

7. Mashiba T, Turner $\mathrm{CH}$, Hirano T, Forwood MR, Johnston CC, Burr DB. Effects of suppressed bone turnover by bisphosphonates on microdamage accumulation and biomechanical properties in clinically relevant skeletal sites in beagles. Bone. 2001;28(5):524-31.

8. Tjong W, Nirody J, Burghardt AJ, Carballido-Gamio J, Kazakia GJ. Structural analysis of cortical porosity applied to HR-pQCT data. Med Phys. 2014;41(1):013701.

9. Viana MP,Tanck E, Beletti ME, Costa Lda F. Modularity and robustness of bone networks. Mol Biosyst. 2009;5(3):255-61.

10. Bernhard A, Milovanovic P, Zimmermann EA, Hahn M, Djonic D, Krause $M$, et al. Micro-morphological properties of osteons reveal changes in cortical bone stability during aging, osteoporosis, and bisphosphonate treatment in women. Osteoporos Int. 2013;24(10):2671-80.

11. Arlot ME, Burt-Pichat B, Roux JP, Vashishth D, Bouxsein ML, Delmas PD. Microarchitecture influences microdamage accumulation in human vertebral trabecular bone. J Bone Miner Res. 2008;23(10):1613-8.

12. Cao L, Moriishi T, Miyazaki T, limura T, Hamagaki M, Nakane $A$, et al. Comparative morphology of the osteocyte lacunocanalicular system in various vertebrates. J Bone Miner Metab. 2011;29(6):662-70.

13. Klein-Nulend J, Nijweide PJ, Burger EH. Osteocyte and bone structure. Curr Osteoporos Rep. 2003;1(1):5-10.

14. Dooley C, Tisbo P, Lee TC, Taylor D. Rupture of osteocyte processes across microcracks: the effect of crack length and stress. Biomech Model Mechanobiol. 2012;11(6):759-66.
15. Sonis ST, Watkins BA, Lyng GD, Lerman MA, Anderson KC. Bony changes in the jaws of rats treated with zoledronic acid and dexamethasone before dental extractions mimic bisphosphonate-related osteonecrosis in cancer patients. Oral Oncol. 2009;45(2):164-72.

16. Rabelo GD, Beletti ME, Dechichi P. Histological analysis of the alterations on cortical bone channels network after radiotherapy: A rabbit study. Microsc ResTech. 2010;73(11):1015-8.

17. de Oliveira RC, Costa LD, Fernandes EA, Alvarenga BOE, Matioli $\mathrm{SR}$, Beletti ME. Bone histomorphometry of broilers submitted to different phosphorus sources in growing and finisher rations. Pesqui Agropec Bras. 2006;41(10):1517-23.

18. Da Fontoura Costa L, Marcondes Cesar R. Shape Analysis and Classification: Theory and Practice. Boca Raton, FL, USA: CRC Press, Inc., 2000.

19. Travençolo BA, Martínez Debat C, Beletti ME, Sotelo Silveira JR, Ehrlich R, Costa LF. A new method for quantifying threedimensional interactions between biological structures. J Anat. 2007;210(2):221-31.

20. Zebaze RM, Ghasem-Zadeh A, Bohte A, luliano-Burns S, Mirams $\mathrm{M}$, Price RI, et al. Intracortical remodelling and porosity in the distal radius and post-mortem femurs of women: a cross-sectional study. Lancet. 2010;375(9727):1729-36.

21. Li B, Ling Chau JF, Wang X, Leong WF. Bisphosphonates, specific inhibitors of osteoclast function and a class of drugs for osteoporosis therapy. J Cell Biochem. 2011;112(5):1229-42.

22. Huja SS, Fernandez SA, Phillips C, LiY. Zoledronic acid decreases bone formation without causing osteocyte death in mice. Arch Oral Biol. 2009;54(9):851-6.

23. Portero-Muzy NR, Chavassieux PM, Bouxsein ML, Gineyts E, Garnero P, Chapurlat RD. Early effects of zoledronic acid and teriparatide on bone microarchitecture, remodeling and collagen crosslinks: comparison between iliac crest and lumbar vertebra in ewes. Bone. 2012;51(4):714-9.

24. Goulet GC, Coombe D, Martinuzzi RJ, Zernicke RF. Poroelastic evaluation of fluid movement through the lacunocanalicular system. Ann Biomed Eng. 2009;37(7):1390-402.

25. Gururaja S, Kim HJ, Swan CC, Brand RA, Lakes RS. Modeling deformation-induced fluid flow in cortical bone's canalicular-lacunar system. Ann Biomed Eng. 2005;33(1):7-25.

26. Himeno-Ando A, Izumi Y, Yamaguchi A, limura T. Structural differences in the osteocyte network between the calvaria and long bone revealed by three-dimensional fluorescence morphometry, possibly reflecting distinct mechano-adaptations and sensitivities. Biochem Biophys Res Commun. 2012;417(2):765-70.

27. Senel FC, Kadioglu Duman M, Muci E, Cankaya M, Pampu AA, Ersoz S, Gunhan O. Jaw bone changes in rats after treatment with zoledronate and pamidronate. Oral Surg Oral Med Oral Pathol Oral Radiol Endod. 2010;109(3):385-91. 